\title{
The effect of warfarin administration on platelet aggregation
}

\author{
Chylova $\mathrm{M}^{1}$, Motovska $\mathrm{Z}^{2}$, Fialova $\mathrm{A}^{3}$, Stetkarova $\mathrm{I}^{1}$, Peisker $\mathrm{T}^{1}$, Kalvach $\mathrm{P}^{1}$ \\ Department of Neurology, Third Faculty of Medicine, Charles University and University Hospital \\ Královské Vinohrady, Prague, Czech Republic. miroslava.chylova@fnkv.cz
}

\begin{abstract}
BACKGROUND: The aim of this study was to assess the effect of anticoagulation treatment on platelet aggregation.

METHODS: The study group consisted of 24 patients on long-term warfarin therapy without any antiaggregation therapy. Platelet aggregation was measured using VerifyNow with arachidonic acid (AA) as an inducer in 23 patients and with light transmission aggregometry (LTA) in 19 patients using four different agonists. All patients had their international normalized ratio (INR) checked regularly.

RESULTS: The mean INR value was 2.07 (SD 0.6). The average aggregation measured by VerifyNow was found to be 637.5 (SD 36.6) aspirin reaction units. The values of average aggregability in LTA were $73.3 \%$ (SD $4.5 \%$ ), $73.2 \%$ (SD $6 \%$ ) and $72.1 \%$ (SD $4.8 \%$ ) in case of aggregation induced by AA, ADP, and collagen, respectively. Epinephrine-induced aggregability was $65.3 \%$ (SD $14.7 \%$ ). Regression analysis between INR and values of collagen- or epinephrine-induced aggregability $(r=0.654$ and 0.575$)$ was found statistically significant $(p=0.004$ and 0.016$)$; every increase in INR by 0,1 brings about an increase in collagen- and epinephrine-induced aggregation values by 1.5 and 4, respectively.

CONCLUSION: Administration of warfarin does not produce a significant reduction in platelet aggregation. On the contrary, prolonged INR evokes a mild increase in aggregation induced by collagen or epinephrine (Tab. 2, Fig. 3, Ref. 32). Text in PDF www.elis.sk

KEY WORDS: platelet aggregation, anticoagulation, warfarin, platelet function tests, chronic ischemic heart disease.
\end{abstract}

\section{Introduction}

Oral anticoagulation with warfarin is recommended in the treatment of venous thromboembolism, as well as in order to reduce the risk of stroke in patients with atrial fibrillation, atrial flutter, or valvular heart disease (1).

Under normal circumstances, proteins including the coagulation factors II, VII, IX, and X require carboxylation by vitamin $\mathrm{K}$ to obtain proper biological activity. Warfarin, a coumarin derivative, induces hepatic production of partially decarboxylated proteins with reduced coagulation activity by inhibiting the vitamin $\mathrm{K}$ conversion cycle (2).

${ }^{1}$ Department of Neurology, Third Faculty of Medicine, Charles University and University Hospital Královské Vinohrady, Prague, Czech Republic, ${ }^{2}$ Department of Cardiology, Third Faculty of Medicine, Charles University and University Hospital Královské Vinohrady, Prague, Czech Republic, and ${ }^{3}$ Department of Biostatistics, National Institute of Public Health, Prague, Czech Republic and Department of Epidemiology and Biostatistics, Third Faculty of Medicine, Charles University, Prague, Czech Republic

Address for correspondence: M. Chýlová, MD, Department of Neurology, Third Faculty of Medicine, Charles University and University Hospital Královské Vinohrady, Šrobárova 50, CZ-100 34 Prague, Czech Republic. Phone: +420 267162380, Fax: +420 267162377

Acknowledgement: This study was supported by a research project at Charles University Progress Q35, UNCE/MED/002, 260533/SVV/2021.
In thrombogenesis two participating processes, namely platelet aggregation and activation of coagulation are closely linked. Despite that, in medical practice, both these particular mechanisms are usually viewed separately and consequently, they are also measured and treated as two isolated categories.

The dichotomy is relevant in patients on anticoagulation or antiaggregation drugs. Considering the tight binding of aggregating thrombocytes to each other via fibrin bridges, we could speculate that any intervention in the coagulation cascade would weaken the mutual adhesion of thrombocytes and thus encumber the efficacy of aggregation.

Haematologic literature contains several observations on the dependency of aggregation on coagulation-suppressing drugs. Such studies have been appearing since the seventies, particularly with heparin, and they reported an induction of increased aggregation along with heparin-induced thrombocytopenia $(3,4,5,6)$, or inhibition of platelet accumulation (7), especially with high doses of heparin (6). More recent studies postulated that warfarin has a stimulating $(8)$ or no effect on platelet aggregation $(9,10)$. However, studies with warfarin are quite rare. The new anticoagulant drugs (NOAC) have been investigated more frequently. Some reveal a reduced platelet aggregability along with supressed coagulation using dabigatran $(11,12,13)$ as well as xabans (14). However, namely with dabigatran, the reports on its effect on aggregability differ. Some authors show an increase (15) while others report no effect $(16,17)$. Beside the human in vitro and ex vivo studies of 
Tab. 1. Baseline characteristics of our patients $\left(n^{1}=24\right)$.

\begin{tabular}{lc}
\hline Mean age (range) & $79(58-92)$ \\
Male/female & $14 / 10$ \\
Arterial hypertension & $11(46 \%)$ \\
Pacemaker or implantable cardioverter defibrillator & $24(100 \%)$ \\
Arrhythmia & $19(79 \%)$ \\
Valvular disease & $6(25 \%)$ \\
Coronary artery disease & $9(38 \%)$ \\
Peripheral arterial disease & $3(13 \%)$ \\
Diabetes mellitus & $8(33 \%)$ \\
Hyperlipidaemia & $16(67 \%)$ \\
Number of thrombocytes & $187.5\left(\mathrm{SD}^{2} 60.6\right) \times 10^{9} / \mathrm{L}$ \\
\hline${ }^{1} \mathrm{n}=$ number of patients ${ }^{2} \mathrm{SD}=$ standard deviation &
\end{tabular}

aggregation influenced by heparin, there are some experimental animal studies carried out in vivo (6).

The comparison of the above-mentioned studies is difficult, since they were carried out with different patient groups, inducers, or aggregometry methods. To obtain our own results of the platelets' readiness to aggregate when influenced with warfarin, we have carried out a study on patients with chronic ischemic heart disease. The purpose of the study was to assess the effect of anticoagulation treatment with warfarin on platelet aggregation in patients with pacemakers or implantable cardioverter defibrillators.

\section{Material and methods}

This study was approved by the ethical committee of the University Hospital Královské Vinohrady, Prague, Czech Republic, and was performed according to the principles of the Declaration of Helsinki. Written informed consent was obtained from all participants.

The platelet aggregation was measured by two different methods, namely by means of bedside optical aggregometry with the VerifyNow system (Accumetrics Inc., San Diego, Calif.) in full blood with arachidonic acid (AA) as an inducer, and by using light transmission aggregometry (LTA) in platelet rich plasma, while using four different agonists - AA, collagen, epinephrine, and adenosine diphosphate (ADP).

The results were expressed in a) aspirin reaction units (ARU) in VerifyNow, where values lower than 550 ARU are considered to be indicative of reduced aggregability (18), and b) LTA as percentage of aggregation (in fact change in light transmission) with $<55 \%$ indicating suppressed aggregation of platelets (19).

All patients were on long-term warfarin therapy with regularly checked INR twice per month. The whole study group consisted of 24 (14 males) patients with pacemakers or implantable cardioverter defibrillators, while being on long-term warfarin therapy and no antiaggregation therapy. Platelet aggregation was measured using VerifyNow in 23 patients and light transmission aggregometry (LTA) was carried out in 19 patients.

The baseline characteristics of the study population are presented in Table 1. No subject had suffered from any recent ischemic event. Users of any other anticoagulant drugs and persons suffering from acute diseases were excluded. The compliance with warfarin intake was ensured by INR levels and face-to-face interviews.

\section{Blood sampling and processing}

Blood for the VerifyNow system was drawn into 2-mL tubes (Greiner Bio-One Vacuette ${ }^{\circledR}$ ) with $3.2 \%$ sodium citrate. The samples were collected atraumatically under standardised conditions using direct venous puncture, while taking care to avoid contamination by tissue factors (free extremity, no retraction). Using a 21-gauge or larger needle, we first collected a volume of at least $2 \mathrm{~mL}$ of blood, which was discarded. We then collected a further volume of $2 \mathrm{~mL}$ of blood in a sample tube. The sample tube was gently inverted at least five times to ensure complete mixing of the contents. Samples with evidence of clotting were not used. After the collection, the samples were maintained at room temperature and then incubated for 30 minutes. All assays were performed according to the manual, i.e., within 2 to $3 \mathrm{~h}$ of sampling (18).

Blood for LTA was drawn into 5-mL tubes containing one part of $3.8 \mathrm{~g} / \mathrm{dl}$ sodium citrate for nine parts of venous blood. The samples were taken in the same manner but we collected a volume of $5 \mathrm{~mL}$ of blood in three sample tubes. Each sample tube was gently inverted at least five times to ensure complete mixing of the contents. After collection, the samples were maintained at room temperature and then incubated for 30 minutes. All assays were performed according to the manual, i.e., within $1 \mathrm{~h}$ of sampling (19).

All platelet aggregation analyses were performed in the morning hours to exclude diurnal changes. We used vacuette tubes containing ethylenediaminetetraacetic acid (EDTA) to measure haemoglobin and platelet counts. Blood for biochemical analyses (especially lipid profile, renal and liver functions, and markers of inflammation) was collected in vacuette tubes without additives, and blood for coagulation analysis was collected in vacuette tubes containing $3.2 \%$ sodium citrate.

\section{Platelet aggregation measurement}

The point-of-care optical aggregometry VerifyNow system (Accumetrics Inc., San Diego, Calif.) was used with arachidonic acid (AA) as an inducer. The results are expressed in aspirin reaction units (ARU). Prior to the insertion into the cartridge that was pre-mounted to the instrument, the blood samples were mixed simply. The aggregation in response to the agonist was monitored by light transmission through two duplicate reaction chambers in each cartridge. Cartridges, routinely used for responses to acetylsalicylic acid (ASA), contained AA as a platelet activator and fibrinogencoated beads. The instrument provides immediate information without any need for transport or delays in special laboratories (20). The results are reported in ARU, with a maximum value of 700 and a minimum value of 350 . Values higher than 550 ARU were indicative of 'no effect' or high on-treatment reactivity, and values below 550 were interpreted as 'reduced aggregability' (18).

Light transmission aggregometry (APACT LPC Software, Version $1.21 \mathrm{c}, \mathrm{Fa}$. LABiTec) was performed with AA, collagen, ADP, and epinephrine as inducers. The results are expressed in percentages. The $100 \%$ line was set equal to platelet-poor plasma (PPP) and the $0 \%$ baseline was established equal to platelet-rich plasma (PRP) prior to the addition of any agonist. PRP was prepared by centrifugation for 15 minutes at $163 \mathrm{~g}$. The aim was to 
Tab. 2. Pearson's correlation coefficient between INR and aggregation using 4 inducers in LTA and using VerifyNow (VN).

\begin{tabular}{lccccc}
\hline & \multicolumn{2}{c}{ INR } & & \multicolumn{2}{c}{ Age } \\
\cline { 2 - 3 } \cline { 5 - 6 } & $\mathrm{R}$ & $P$-value & & $\mathrm{R}$ & $P$-value \\
\hline ADP & 0.450 & 0.070 & & 0.201 & 0.440 \\
Collagen & 0.654 & $0.004^{2}$ & & -0.059 & 0.821 \\
Epinephrine & 0.575 & $0.016^{1}$ & & -0.097 & 0.710 \\
AA & 0.425 & 0.089 & & 0.011 & 0.966 \\
VN & 0.110 & 0.636 & & -0.304 & 0.181 \\
\hline
\end{tabular}

${ }^{1}$ significant at the 0.05 level, ${ }^{2}$ significant at the 0.01 level

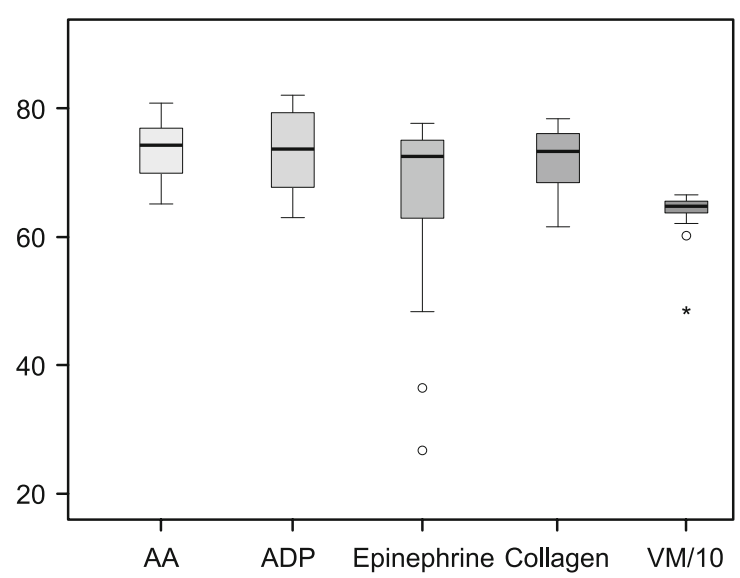

Fig. 1. Level of aggregation in patients on long-term warfarin therapy. Results of LTA using four different agonists (AA, ADP, epinephrine and collagen) are shown as percentage of light transmission. Those of VerifyNow (VerifyNow Measurements in ARUs/10 $=\mathrm{VM} / \mathbf{1 0}$ ) are in ARU units.

concentrate the platelets to values recommended in the literature, i.e., between 200 and $400 \times 109 / 1(19,21)$. PPP was prepared by further centrifugation for 15 minutes at $2500 \mathrm{~g}$. For very high counts, the specimen was adjusted to optimum by PPP. PRP was stirred in $200 \mu \mathrm{L}$ minicuvettes at $37.4{ }^{\circ} \mathrm{C}$ and each cuvette was placed between the light source and photocell. The addition of a platelet agonist to PRP provokes the platelet activation and brings about a change in their shape from discoid to spiny spheres, while reducing the absorption of light by platelets. After one of the four agonists mentioned above is added, the platelets aggregate and thus absorb less light, which increases the transmission detected by the photocell $(19,20)$. Four different inducers, namely AA, collagen, epinephrine, and ADP (in final concentrations of $0.5 \mathrm{mg}$ / $\mathrm{mL}, 10 \mu \mathrm{g} / \mathrm{mL}, 10 \mu \mathrm{mol} / \mathrm{L}$, and $20 \mu \mathrm{mol} / \mathrm{L}$, respectively) were individually added. The shape of the curves and percentage of aggregation were recorded $(19,22,23,24,25)$. In LTA, the limit of sufficient reduction of aggregation after treatment was set to the value of $<55 \%$ according to literature, where such values are broadly reported for all agonists. These limits are in agreement with the limits of our laboratory.

\section{Statistical analysis}

The first statistical step was the comparison of the whole averaged cohorts in particular aggregometry methods. Next, we per- formed regression models to test the linear relationship between aggregation using four different inducers as dependent variables and INR as an independent variable. Prior to statistical testing, the Dean-Dixon test was used to detect outliers in a univariate data set. Furthermore, the age of patients was included as a second regressor in the models. The Pearson correlation coefficient $r$ and coefficient of determination R2 were used to quantify the degree of association. ANOVA and t-test were used to test the significance of linear regression model. The statistical significance was set to $5 \%$. The data were processed using IBM SPSS Statistics for Windows, version 24 (IBM Corp., Armonk, N.Y., USA).

\section{Results}

All of our patients had effective INR levels. The mean INR value was 2.07 (SD 0.6). The mean platelet aggregation in patients on long-term warfarin therapy was 637.5 (SD 36.6) ARU using VerifyNow. The mean aggregability values in LTA with AA, ADP and collagen were $73.3 \%$ (SD $4.5 \%$ ), $73.2 \%$ (SD 6) and $72.1 \%$ (SD 4.8), respectively. Epinephrine-induced aggregability was $65.3 \%$ (SD 14.7). Thus, these values did not reach the limits of our laboratory in terms of a significantly reduced standard aggregation (Fig. 1).

To decipher the relation between anticoagulation and aggregability in greater detail, we have performed regression analysis for individual persons. While in VerifyNow and on arachidonic acid or ADP as inducers, no significant correlation was found, the aggregation induced by collagen, and even more that induced by epinephrine appeared to be significantly dependent on warfarin (Tab. 2).

The model explains $42.7 \%$ and $33.1 \%$ of data variability by the use of collagen and epinephrine inducers, respectively. The data indicate that every increase in INR by 0.1 results in a statistically significant increase in the collagen-induced aggregation by $1.5(p=0.004)$. Similarly, an INR increase by 0.1 is accompanied by an increase in epinephrine-induced aggregability by 4.0 ( $\mathrm{p}=$ 0.016) (Figs 2 and 3).

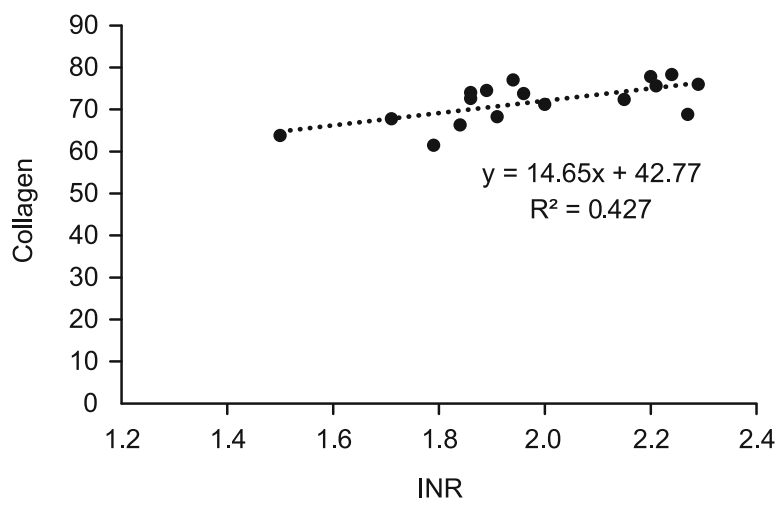

Fig. 2. Linear regression relationship between collagen and INR. Results of LTA using collagen as agonist are shown as percentage of light transmission. INR values are plotted on the $x$ axis. 


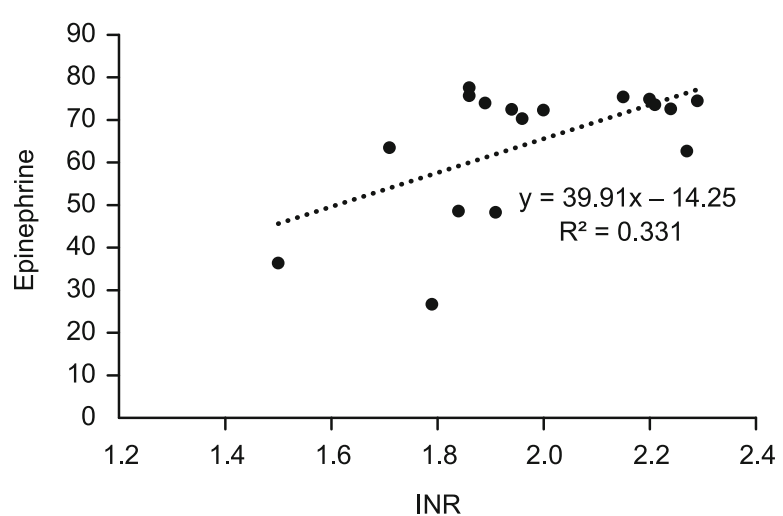

Fig. 3. Linear regression relationship between epinephrine and INR. Results of LTA using epinephrine as agonist are shown as percentage of light transmission. INR values are plotted on the $x$ axis.

\section{Discussion}

Already eighty years have elapsed from the time when the scientists started questioning the relation between the two main components of blood clotting, namely coagulation and platelet aggregation (26). Yet, during that time, the interconnection of these mutually dependent and intertwined processes has not been disentangled. In medical practice, coagulation is usually measured separately and more reliably as an aggregation of thrombocytes.

It is well known from meta-analytic studies that adjusted doses of warfarin and antiplatelet agents have reduced the risk of stroke in patients with atrial fibrillation by approximately $60 \%$ and approximately $20 \%$, respectively (27). Many patients with atrial fibrillation and ischemic heart disease who are treated with anticoagulation agents also need antiaggregation treatment due to other diseases such as atherothrombosis as well as due to the risks of ischemic heart disease or stroke. We usually do not add antiaggregation treatment in these patients due to the potential risks of serious bleeding complications. In this study, we were interested if the platelet aggregation in these anticoagulated patients would be influenced as well and if we would be able to measure this effect.

Our previous experience has taught us that aggregometry is not a simple and one-way process $(28,29)$. The methods do not measure any spontaneous run of changes, but depend on the induction of aggregation by its particular stimulants. We were interested in finding whether the warfarin-induced haemocoagulation would entail a simultaneous measurable derangement of aggregation provoked by several particular inductors. For this reason, in persons on warfarin, we have analysed aggregometry with LTA using AA, ADP, collagen and epinephrine and by point-of-care test VerifyNow with AA as agonist.

The results of the whole group when presented as average were similar to the aggregation values in healthy individuals without any antiagregation or anticoagulation treatment $(28,29)$. Administration of warfarin does not produce a significant reduction in platelet aggregation. Similar results were described previously $(30,31)$.

After we had deployed the regression model of intraindividual comparison, some correlation became apparent. In case of aggre- gation induction by collagen and mainly by epinephrine, a clear effect of prolonged INR on the increased aggregability could be registered. It seems that because these pathways serve as a rescue mechanism in case of stress or insult to the vessel wall, not only the aggregation is unaffected, it increases even despite warfarin therapy. In case of aggregation induction by ADP and arachidonic acid, no substantial dependence was found; these agonists are in play for different drugs' pathways, and in warfarin patients, we were not able to find significant correlations.

We assume that platelet aggregation is spurred by manifold pathways. The use of different agonists in experiment provides disparate results. This may have caused inconsistent findings in our own study, as well as in a number of similar experiments in literature. It is possible that using the thrombin receptor-activating peptide (TRAP) as the most potent agonist of platelet activation via protease-activated receptors 1 (PAR1) and 4 could give more reliable data. If so, it would correspond with studies on NOAC's. We may hypothesise that the use of TRAP as an agonist in LTA could help us to detect the effect on platelet aggregation with warfarin as well. Moreover, it is clear that the choice of a reliable and verified method is paramount because the ability to detect a change in aggregation can vary a lot (32).

We are aware of the fact that aggregability tests do not fully correspond with the global aggregation in vivo. What we could measure and investigate was always only one particular pathway with one concrete inducer, or one concrete receptor. The complex system of induction and suppression of aggregation allows many ways of aggregation to remain unaffected, thus precluding the detection of some particular changes in some pathways. We cannot say with certainty if the suppression in one pathway can be stronger than in others.

\section{Conclusion}

Contrary to our original hypothesis, we were not able to demonstrate the reducing effect of anticoagulation on aggregability using LTA or VerifyNow. The administration of warfarin did not produce a significant reduction in platelet aggregation. The correlation of INR with the results of aggregation induced by collagen and epinephrine turned out to be not on line with our expectations. Every increase in INR means a mild increase in aggregation of platelets, thus expressing only a minimal effect of warfarin on these pathways. The effect of warfarin on aggregation was not comprehensive; some ways of aggregation induction appeared uninfluenced.

Since the aggregability depends on the applied inducer, we speculate that the application of thrombin receptor-activating peptide (TRAP) as an agonist in LTA could eventually bring more consistent results.

\section{References}

1. Wigle P, Hein B, Bloomfield HE, Tubb M, Doherty M. Updated Guidelines on Outpatient Anticoagulation. Am Fam Physician 2013; 87 (8): 556-566. 
2. Hirsh J, Fuster V, Ansell J and Halperin JL. American Heart Association/American College of Cardiology Foundation Guide to Warfarin Therapy. Circulation 2003; 107: 1692-1711.

3. Hussey CV, Bernhard VM, McLean MR, Fobian JE. Heparin induced platelet aggregation: in vitro confirmation of thrombotic complications associated with heparin therapy. Ann Clin Lab Sci 1979; 9 (6): 487-493.

4. Demasi R, Bode AP, Knupp C, Bogey W, Powell S. Heparin-induced thrombocytopenia. Am Surg 1994; 60 (1): 26-29.

5. Frick G, Frick U, Feyerherd F. Behavior of thrombocytes in lowdose heparin therapy. Folia Haematol Int Mag Klin Morphol Blutforsch 1987; 114 (5): 656-660.

6. Barrett PA, Butler KD, Morley J, Page CP, Paul W, White AM. Inhibition by heparin of platelet accumulation in vivo. Thromb Haemost 1984; 51: 366-370.

7. John LCH, Rees GM, Kovacs IB. Inhibition of platelet function by heparin. J Thoracic Cardiovasc Surg 1993; 105 (5): 816-822.

8. Mieszczak C, Winther K. Does warfarin enhance platelet activity? Thromb Res 1996; 84: 285-287.

9. Ferreira CN, Vieira LM, Dusse LMS et al. Evaluation of the blood coagulation mechanism and platelet aggregation in individuals with mechanical or biological heart prostheses. Blood Coagul Fibrinolysis 2002; 13 (2): 129-134.

10. Kawahito K, Adachi H, Ino T. Platelet aggregation in patients taking anticoagulants after valvular surgery: evaluation by a laser light-scattering method. Artif Organs 2002; 5 (3): 188-192.

11. Sokol J, Nehaj F, Ivankova J, Mokan M, Mokan M, Stasko J. Dabigatran affects thrombin-dependent platelet aggregation after a week-long therapy. Scand Cardiovasc J 2018; 52 (4): 227-231.

12. Nehaj F, Sokol J, Mokan M, Ivankova J, Mokan M. Thrombin receptor agonist peptide-induced platelet aggregation is reduced in patients receiving dabigatran. Clin Appl Thromb Hemost 2018; 24 (2): 268-272.

13. Shimizu M, Natori T, Tsuda K et al. Thrombin-induced platelet aggregation -effect of dabigatran using automated platelet aggregometry. Platelets $2020 ; 31$ (3): 360-364.

14. Nehaj F, Sokol J, Ivankova J et al. First Evidence: TRAP - induced platelet aggregation is reduced in patients receiving xabans. Clin Appl Thomb Hemost 2018; 24 (6): 914-919.

15. Olivier CB, Weik P, Meyer M et al. TRAP-induced platelet aggregation is enhanced in cardiovascular patients receiving dabigatran. Thromb Res 2016; 138: 63-66.

16. Martischnig AM, Mehilli $\mathbf{J}$, Pollak $\mathbf{J}$ et al. Impact of dabigatran versus phenprocoumon on ADP induced platelet aggregation on patients with atrial fibrillation with or without concomitant clopidogrel therapy (the Dabi-ADP-1 and Dabi-ADP-2 Trials). Biomed Res Int 2015: 798486.
17. Olivier CB, Weik P, Meyer M et al. Dabigatran and rivaroxaban do not affect AA- and ADP-induced platelet aggregation in patients receiving concomitant platelet inhibitors. J Thromb Thrombolysis 2016; 42 (2): 161-166.

18. User Manual, Accumetrics Inc., USA. VerifyNow System 2006; 1-122.

19. http://www.practical-haemostasis.com/Platelets/platelet_function_testing_Ita.html

20. Harrison P, Frelinger AL3rd, Furman MI, Michelson AD. Measuring antiplatelet drug effects in the laboratory. Thromb Res 2007; 120: 323-336.

21. Merolla M, Nardi MA, Berger JS. Centrifugation speed affects light transmission aggregometry. Int J Lab Hematol 2012; 34(1): 81-85.

22. Harrison P, Segal H, Blasbery K, Furtado C, Siver L, Rothwell PM. Screening for aspirin responsiveness after transient ischemic attack and stroke: comparison of 2 point-of-care platelet function tests with optical aggregometry. Stroke 2005; 36 (5): 1001-1005.

23. Gum PA, Kottke-Marchant K, Welsh PA, White J, Topol EJ. A prospective, blinded determination of the natural history of aspirin resistance among stable patients with cardiovascular disease. J Am Coll Cardiol 2003; 41 (6): 961-965.

24. APACT 4004, four chanels aggregometer for assesing aggregation of platelets in vitro, user manual, Biogenix s.r.o., Prague, Czech Republic.

25. Hayward CP, Moffat KA, Raby A et al. Development of North American consensus guidelines for medical laboratories that perform and interpret platelet function testing using light transmission aggregometry. Am J Clin Pathol 2010; 134 (6): 955-963.

26. Solandt DY, Best CH. Time relation of heparin action on blood clotting and platelet agglutination. Lancet 1940; 1: 1042.

27. Hart RG, Pearce LA, Aguilar MI. Meta-analysis: antithrombotic therapy to prevent stroke in patients who have nonvalvular atrial fibrillation. Ann Intern Med 2007; 146 (12): 857-867.

28. Chýlová M, Mot'ovská Z, Osmančík P, Procházka B, Kalvach P. The effect of different doses and different routes of acetylsalicylic acid administration on platelet aggregation in healthy volunteers and ischemic stroke patients. Transl Stroke Res 2015; 6 (2): 160-165.

29. Chýlová M, Kejst L, Procházka B, Kalvach P. Combination of two Optic Platelet Aggregometry Methods in Detection of Aspirin Effectivity. Exp Clin Cardiol 2014; 20: 800-811.

30. Ho LL, Brighton T. Warfarin, antiplatelet drugs and their interactions. Australian Prescriber 2002; 25 (4): 81-84.

31. Helgason CM, Hoff JA, Kondos GT, Brace LD. Platelet aggregation in patients with atrial fibrillation taking aspirin or warfarin. Stroke 1993; $24: 1458-1461$.

32. Eller T, Busse J, Dittrich M et al. Dabigatran, rivaroxaban, apixaban, argatroban and fondaparinux and their effects on coagulation POC and platelet function tests. Clin Chem Lab Med 2014; 52 (6): 835-844.

Received October 11, 2020. Accepted October 25, 2020. 